\title{
Bayesian Estimation of Spatio-Temporal Models with Covariates Measured with Spatio-Temporally Correlated Errors: Evidence from Monte Carlo Simulation
}

\author{
Mohammad Masjkur ${ }^{1}$, Henk Folmer ${ }^{2}$ \\ ${ }^{1}$ Department of Statistics, Faculty of Mathematics and Natural Sciences, Bogor Agricultural University, Indonesia \\ ${ }^{2}$ Faculty of Spatial Sciences, University of Groningen, The Netherlands \\ masjkur@apps.ipb.ac.id, h.folmer@rug.nl
}

\begin{abstract}
Spatio-temporal data are susceptible to covariates measured with errors. However, little is known about the empirical effects of measurement error on the asymptotic biases in regression coefficients and variance components when measurement error is ignored. The purpose of this paper is to analyze Bayesian inference of spatio-temporal models in the case of a spatio-temporally correlated covariate measured with error by way of Monte Carlo simulation. We consider spatio-temporal model with spatio-temporal correlation structure corresponds to the Leroux conditional autoregressive (CAR) and the first order autoregressive priors. We apply different spatio-temporal dependence parameter of response and covariate. We use the relative bias (RelBias) and Root Mean Squared Error (RMSE) as valuation criteria. The simulation results show the Bayesian analysis considering measurement error show more accurate and efficient estimated regression coefficient and variance components compared with naïve analysis.
\end{abstract}

Keywords Spatio-temporal model, measurement error, Bayesian analysis

\section{Introduction}

Space-time data are common in social sciences, epidemiology, environmental and agricultural sciences. The data are typically collected from points or regions located in space and over time. That sample data commonly observed are not independent, but rather spatially and temporally dependent, which means that observation from one location-time tend to exhibit values similar to those from nearby locations-time. Ignoring the violation of spatial and temporal independence between observations will produce estimates that are biased and inconsistent.

A large variety of spatio-temporal models to take spatio-temporal dependence among observations into account have been developed (Rushworth et al., 2014; Ugarte et al., 2014; Truong et al., 2016). An approach is the mixed effects model which modeling the random effects of the spatial and temporal correlations structure.

Spatio-temporal data are susceptible to covariates measured with errors. Li et al. (2009) showed that the estimator of the regression coefficients are attenuated, while the estimator of the variance components are inflated, if covariate measurement error is ignored. Furthermore, Huque et al. (2014) showed that the amount of attenuation depends on the degree of spatial correlation in both the true covariate of interest and the assumed random error from the regression model.

Several approaches to correct for measurement error have been proposed in literature for independent data (Muff et al., 2015; Stoklosa et al., 2016). However, limited work has been done in modeling measurement error in covariates for spatio-temporal data. For spatial data, Li et al. (2009) proposed the use of maximum likelihood based on EM algorithm to adjust for measurement error under the assumed correlation structure. The estimators of the regression coefficients and the variance components correct the biases in naive estimator and have smaller MSE than the naïve estimators. However, their simulation assumes that the measurement error variance is known. Huque et al. (2014) proposed two different strategies to produce consistent estimates: (i) adjusting the estimates using an estimated attenuation factor, and (ii) using an appropriate transformation of the error prone covariate. Additionally, Huque et al. (2016) proposed a semiparametric approach to obtain bias-corrected estimates of parameters. They used penalized least squares which makes the estimation of parameters and inference straightforward.

For spatio-temporal data, Xia and Carlin (1998) presented a spatio-temporal analysis of spatially correlated data accounted for measurement error in covariates using Gibbs sampling. However, little is known about the empirical effects of measurement error on the asymptotic biases in re- 
gression coefficients and variance components when measurement error is ignored.

Muff et al. (2015) stated that among several approaches to correct for measurement error, Bayesian methods probably provide the most flexible framework. The advantage of Bayesian approaches is that prior knowledge, and in particular prior uncertainty of error variance estimates can be incorporated in the model. While frequentist approaches require to fix the regression coefficients and the variance components parameters to guarantee identifiability, the Bayesian setting allows to represent uncertainty with suitable prior distributions.

The purpose of this paper is to analyze Bayesian inference of spatio-temporal models in the case of a spatio-temporally correlated covariate measured with error by way of Monte Carlo simulation.

\section{Regression Model with Measurement Error}

Muff et al. (2015) presented the framework of generalized linear (mixed) model with measurement error (ME) as follows,

\subsection{The Generalized Linear (Mixed) Model}

Let $y=\left(y_{1} \ldots \ldots \ldots y_{n}\right)^{T}$ be the observable response variable collected from site $i=1, \ldots ., n$ which is related to some set of $\mathrm{k}$ error free covaraites $\boldsymbol{z}=\left(z_{1, \ldots \ldots \ldots} z_{k}\right)$ and a single error prone true and unobservable covariate $\boldsymbol{x}=\left(x_{1, \ldots \ldots \times x} x_{n}\right)^{T}$. Suppose that $\mathrm{y}$ is of exponential family form with mean $\mu_{i}=E\left(y_{i} \mid x_{i}\right)$ linked to the linear predictor $\eta_{i}$ with

$$
\begin{aligned}
& \mu_{i}=h\left(\eta_{i}\right), \\
& \eta_{i}=\beta_{0}+\beta_{x} x_{i}+z_{\text {「in }} \beta_{z}
\end{aligned}
$$

Here, $\mathrm{h}($.$) is a known monotonic inverse link (or response)$ function, $\beta_{0}$ the intercept, $\beta_{x}$ the fixed effect for the error prone covariate $\mathrm{x}$ and $\mathrm{z}_{\text {[în }}$ is $1 \mathrm{x} \mathrm{k}$ with a corresponding vector $\beta_{z}$ of fixed effects. This generalized linear model is extended to a generalized linear mixed model by adding normally distributed random effects on the linear predictor scale (1).

\subsection{Classical Measurement Error Model}

Let $p=\left(p_{1} \ldots \ldots \ldots p_{n}\right)^{T}$ denote the observed version of the true, but unobserved covariate $x$. In the classical measurement error model it is assumed that the covariate $\mathrm{x}$ can be observed only via a proxy $\mathrm{p}$, such that in vector notation,

$$
\mathrm{p}=\mathrm{x}+\mathrm{u} \text {, }
$$

with $\boldsymbol{u}=\left(u_{1, \ldots \ldots n} u_{n}\right)^{T}$. The components of the error vector $\mathrm{u}$ are assumed to be independent and normally distributed with mean 0 and variance $\tau_{\mathcal{u}}^{-1}$, i.e. $\operatorname{cov}\left(u_{i}, u_{j}\right)=0$ for $\mathrm{i} \neq \mathrm{j}$. The error structure can be heteroscedastic with $\boldsymbol{p}_{\tilde{i}} \sim \mathbb{N}\left(\boldsymbol{x}_{v} \tau_{\mathrm{u}} \boldsymbol{D}\right)$, where the elements in the diagonal matrix $D$ represent known weight $\mathrm{d}_{\mathrm{i}}>0$.

In the most general case, the covariance $\mathrm{x}$ is Gaussian with mean depending on $z$, i.e.

$$
x \mid z \sim N\left(\alpha_{0} 1+z \alpha_{z}, \tau_{x} I\right)
$$

where $\alpha_{0}$ is the intercept, $\boldsymbol{\alpha}_{z}$ the k x 1 vector of fixed effects and $\tau_{x}^{-1}$ the residual variance in the linear regression of $\mathrm{x}$ on $\mathrm{z}$. If $\alpha_{z}=0$, then $\mathrm{x}$ is independent of $\mathrm{z}$.

The latent Gaussian hierarchical model for classical measurement error (ME) model defined as follows,

(i) The observational model encompasses two components, namely the regression model and the error model:

$$
\begin{aligned}
& E(y \mid x)=h\left(\beta_{0} 1+\beta_{x} x+z \beta_{z}\right), \\
& \mathrm{p}=\mathrm{x}+\mathrm{u}, u \sim N\left(0, \tau_{u} D\right)
\end{aligned}
$$

$\mathrm{p}$ is now part of the observational model, which is thus $\mathrm{y}, \mathrm{p} \mid \mathrm{v}$, $\theta_{1}$ instead of $y \mid v, \theta_{1}$.

(ii) The latent part contains the exposure model for $\mathrm{x}$

$$
x=\alpha_{0} 1+z \alpha_{z}+\epsilon_{x}, \epsilon_{x} \sim N\left(0, \tau_{x} I\right),
$$

as well as the specification of independent Gaussian priors for the regression coefficients. Thus the latent field is

$$
\mathrm{v}=\left(\mathrm{x}^{\mathrm{T}}, \beta_{0}, \beta_{\mathrm{z}}^{\mathrm{T}}, \alpha_{0}, \alpha_{\mathrm{z}}^{\mathrm{T}}\right)^{\mathrm{T}} \text {. }
$$

The exposure model (2) can be extended to include structured or unstructured random effects.

(iii) The third level describes the prior distributions for all hyperparameters

$$
\boldsymbol{\theta}=\left(\beta_{\mathrm{x}}, \tau_{\mathrm{u}}, \tau_{\mathrm{x}}, \boldsymbol{\theta}_{1}^{\mathrm{T}}\right)^{\mathrm{T}},
$$

with $\theta_{1}$ representing (possible) hyperparameters of the likelihood. The regression coefficient $\beta_{\mathrm{x}}$ is also considered as an unknown hyperparameter, and not as part of the latent field. The following priors were considered, i.e., the normal prior with mean 0 and low precision for $\beta_{\mathrm{x}}$ and gamma priors for $\tau_{\mathrm{x}}$ and $\tau_{\mathrm{u}}$.

\section{Simulation}

We consider the spatio-temporal model (location $i$ and time $t$ ) with a single true covariate $X_{\text {it }}$ as follows:

$$
Y_{i t}=\beta_{0}+X_{i t} \beta_{x}+\varphi_{i t}+\epsilon_{i t}
$$

with $Y_{\text {it }}$ the response in location $i\left(i=1_{x \ldots,} N\right)$ during time period $t\left(t=1_{x \ldots} T\right) ; X_{i t}$ is an unobserved true covariates relating to location $i$ during time period $t, \beta_{x}$ is the associated regression parameter of $X_{i t}, \varphi_{i t}$ are the random effects after the effects of covariate has been removed that are spatio-temporally correlated and $\epsilon_{i t}$ is the residual $\sim N\left(0, \sigma_{e}^{2}\right)$ (Rushworth et al., 2014; Truong et al., 2016).

The random effects $\varphi_{\text {it }}$ defined as follows

$$
\begin{aligned}
& \varphi_{t 1} \mid \varphi_{-t 1} \sim N\left(\frac{\rho_{s} \sum_{j=1}^{n} w_{i j} \varphi_{j 1}}{\rho_{s} \sum_{j=1}^{n} w_{i j}+1-\rho_{s}}, \frac{\tau^{2}}{\rho_{s} \sum_{j=1}^{n} w_{i j}+1-\rho_{s}}\right) \\
& \varphi_{t} \mid \varphi_{t-1} \sim N\left(\rho_{T} \varphi_{t-1} \tau^{2} Q\left(W_{s} \rho_{s}\right)^{-1}\right) t=2{ }_{s \ldots \ldots} T
\end{aligned}
$$

where $\varphi_{-i 1}$ is the random effects for time period 1 except for $\varphi_{\mathrm{i} 1}, \varphi_{\mathrm{t}}$ is the vector of random effects for time period $t, W=\left\{w_{i f}\right\}$ is the $n x n$ adjacent matrix ( $w_{i f}=1$ if areas $i$ and $\hat{i}$ are adjacent or 0 otherwise), $P_{5}$ is the spatial parameter, $\rho_{T}$ is the temporal parameter, and $\tau^{2}$ is the parameter controlling the variance of random effects. The precision matrix $Q\left(W_{,}, \rho_{5}\right)$ corresponds to the Leroux conditional autoregressive (CAR) prior and is given by $\left.Q\left(W_{s} \rho_{s}\right)=\rho_{s}\left(\operatorname{diag} W 1_{n}\right)-W\right)+\left(1-\rho_{s}\right) I$, where $1_{n}$ is the $n x 1$ vector of ones, $I$ is the $\mathrm{n} \times \mathrm{n}$ identity matrix.

We assume a spatio-temporal random effects model for the unobserved covariate $X$ : 


$$
X_{i t}=a_{0}+a_{i t}+e_{i t}
$$

where $a_{i t}$ are random effects for spatio-temporal autocorrelation in the covariate $\mathrm{X}$ and $e_{\text {it }}$ is the residual $\sim N\left(0, \sigma_{\theta}^{2}\right)$ similar to (1) with different parameter.

We assume that $P_{\text {it }}=X_{\text {it }}+U_{\text {it }}$, where $P_{\text {it }}$ is the observed covariates related to the true covariates $X_{\text {it }}$ according to a classical measurement error model with $U_{i t} \sim N\left(0, \sigma_{U}^{2}\right)$.

We take the data to be on a regular grid. The weight $\mathrm{w}_{\mathrm{ij}}$ is set to be 1 if areas $i$ and $\mathrm{j}$ are neighbors and 0 otherwise. The spatial dependence parameter for $\mathrm{X}$ is considered to be $P_{5 x}=0.1,0.5,0.9$ resulting in minimal, moderate and high correlation. The variance parameter for space-time interaction and residual error term are taken as 0.3 and 0.1 , respectively. We consider the temporal dependences parameter $\rho_{T x}=0.5$ and 0.9 respectively. The observed error-prone covariate $P$ is generated by adding Gaussian noise with variance $\sigma_{U}^{2}=0.3$ to $X$. Outcome data, $Y$, are then generated according to equation (6), with slope and intercept parameters set at $\left(\beta_{0}, \beta_{x}\right)^{\mathrm{T}}=(1,2)^{\mathrm{T}}$. The variance parameter for space-time interaction and residual error term are taken as 0.2 and 0.1 , respectively. The spatial dependence taken to be 0.5 and the temporal dependences parameter similar to $X$. We consider the grid size to be $7(n=7 \times 7)$ and $10(\mathrm{n}=10 \times 10)$, and $\mathrm{T}=10$ consecutive time period.

We generate 100 Monte Carlo simulation datasets. For each generated dataset, we compute the Bayesian estimates that ignored (naïve estimates) and accounted for the measurement error, respectively.

We compute the relative bias (RelBias) and the Root Mean Square Error (RMSE) for each parameter estimate over 100 samples for each simulation. These statistics are defined as

$$
\operatorname{RelBias}(\theta)=\frac{1}{k} \sum_{j=1}^{k}\left(\frac{\widehat{\theta}_{j}}{\theta}-1\right), \quad \operatorname{RMSE}(\theta)=\sqrt{\frac{1}{k} \sum_{j=1}^{k}\left(\hat{\theta}_{j}-\theta\right)^{2}}
$$

where $\widehat{\theta}_{i}$ is the estimate of $\theta$ for the $j^{\text {th }}$ sample and $\mathrm{k}=100$.

We also compare the models based on Marginal Log-Likelihood, Deviance Information Criterion (DIC), and Watanabe-Akaike Information Criterion (WAIC). These statistics are defined as

$\overline{D I C}=\overline{\mathcal{D}}+2 p_{D I C}$ and $\widehat{W A I C}=-2$ lppd +2 PWAIC where $\bar{D}=\sum_{n=1}^{Q} \mathcal{D}\left(\theta_{\alpha}\right) / Q$ the posterior mean of the deviance, $\mathcal{D}(\boldsymbol{\theta})=-2 \sum_{i=1}^{n} \log \left\{f\left(y_{i} \mid \boldsymbol{\theta}\right)\right\}$, which $\left\{f\left(y_{i} \mid \boldsymbol{\theta}\right)\right\}$ the likelihood function, and $Q$ is the number of iterations, lppd the log pointwise predictive density, and $p$ the effective number of parameters (Gelman et al., 2014).

We fitted the models using the INLA R-package available at http://www.r-inla.org. We consider independent Gaussian $\mathrm{N}\left(0,10^{-4}\right)$ prior to regression coefficient $\beta_{\mathrm{x}}$, and gamma $\mathrm{G}(0.01,0.01)$ priors to the precision parameter $\tau_{\mathrm{u}}, \tau_{\mathrm{x}}$, and $\tau_{\varepsilon}$.

\section{Main Results}

Table 1 and 2 show that the degree of RelBias and RMSE for regression coefficients for measurement error and naïve models vary with the strength of the spatial and temporal correlation structure of covariate as well as the residuals.
However, the average RelBias (in absolute value) and the average RMSE for regression coefficients of the measurement error model smaller than the naïve model.

Note that both methods underestimate the true regression coefficient $\beta_{x}$ and increase with the spatial dependence parameter of covariate. For naïve model, the average RelBias (in absolute value) for regression coefficients $\beta_{x}$ decrease with the temporal dependence parameter, but increase for measurement error model. Note that the temporal dependence parameter of response and covariate are the same. However, the measurement error model estimator's consistently provides less bias compared with the naïve model.

The average RelBias (in absolute value) and the average $R M S E$ for variance components of the measurement error model also smaller than the naïve model. Note that the average RelBias for spatial variance components $\sigma_{\text {sy }}^{2}$ of both methods increase with the spatial and temporal dependence parameter. According to $\mathrm{Li}$ et al. (2009) and Huque et al. (2014; 2016) that naïve estimator of regression coefficient attenuated and the variance components inflated if covarate measurement error ignored. Furthermore, Li et al. (2009) stated that the stronger dependence implies that neighbor areas can provide more information, and hence the estimates are more resistant to the effect of measurement error.

Table 1. RelBias and RMSE of Regression Coefficients and Variance Components for Bayesian Spatio-Temporal Measurement Error and Naïve

\begin{tabular}{|c|c|c|c|c|c|c|}
\hline \multicolumn{7}{|c|}{ Model } \\
\hline \multirow[t]{2}{*}{$\rho_{\mathrm{T}}$} & \multirow[t]{2}{*}{$\left(\rho_{\mathrm{sy}}, \rho_{\mathrm{sx}}\right)$} & \multirow{2}{*}{$\begin{array}{l}\text { Param- } \\
\text { eter }\end{array}$} & \multicolumn{2}{|l|}{$\mathrm{ME}$} & \multicolumn{2}{|l|}{ NAIVE } \\
\hline & & & RelBias & $R M S E$ & RelBias & $R M S E$ \\
\hline \multirow[t]{12}{*}{0.5} & $(0.5,0.1)$ & $\beta_{0}$ & 0.0103 & 0.0757 & 0.0102 & 0.0755 \\
\hline & & $\beta_{\mathrm{x}}$ & -0.0412 & 0.2555 & -0.4529 & 0.9079 \\
\hline & & $\sigma_{\text {sy }}^{2}$ & 0.8724 & 0.2931 & 1.2371 & 0.3453 \\
\hline & & $\sigma_{\varepsilon}^{2}$ & -0.2935 & 0.0572 & 5.6874 & 0.5851 \\
\hline & $(0.5,0.5)$ & $\beta_{0}$ & -0.0158 & 0.0858 & -0.0162 & 0.0861 \\
\hline & & $\beta_{\mathrm{x}}$ & -0.1064 & 0.3423 & -0.5261 & 1.0544 \\
\hline & & $\sigma_{\text {sy }}^{2}$ & 1.5012 & 0.3894 & 1.9092 & 0.4738 \\
\hline & & $\sigma_{\varepsilon}^{2}$ & -0.3875 & 0.0563 & 4.1544 & 0.4430 \\
\hline & $(0.5,0.9)$ & $\beta_{0}$ & 0.0087 & 0.1678 & 0.0078 & 0.1623 \\
\hline & & $\beta_{\mathrm{x}}$ & -0.2904 & 0.6418 & -0.5184 & 1.0422 \\
\hline & & $\sigma_{\text {sy }}^{2}$ & 3.3781 & 0.7326 & 2.7439 & 0.7406 \\
\hline & & $\sigma_{\varepsilon}^{2}$ & -0.2451 & 0.0449 & 3.6150 & 0.4645 \\
\hline \multirow[t]{12}{*}{0.9} & $(0.5,0.1)$ & $\beta_{0}$ & 0.0087 & 0.1465 & 0.0087 & 0.1465 \\
\hline & & $\beta_{\mathrm{x}}$ & -0.2315 & 0.4712 & -0.4333 & 0.8689 \\
\hline & & $\sigma_{\text {sy }}^{2}$ & 7.4841 & 1.5411 & 7.6420 & 1.5740 \\
\hline & & $\sigma_{\varepsilon}^{2}$ & -0.5994 & 0.0661 & 4.6885 & 0.4744 \\
\hline & $(0.5,0.5)$ & $\beta_{0}$ & 0.0150 & 0.1852 & 0.0150 & 0.1851 \\
\hline & & $\beta_{\mathrm{x}}$ & -0.2998 & 0.6096 & -0.5216 & 1.0452 \\
\hline & & $\sigma_{\text {sy }}^{2}$ & 6.9942 & 1.4232 & 7.1501 & 1.4540 \\
\hline & & $\sigma_{\varepsilon}^{2}$ & -0.4383 & 0.0642 & 3.6338 & 0.3696 \\
\hline & $(0.5,0.9)$ & $\beta_{0}$ & -0.0054 & 0.3590 & -0.0055 & 0.3590 \\
\hline & & $\beta_{\mathrm{x}}$ & -0.3632 & 0.7339 & -0.5339 & 1.0695 \\
\hline & & $\sigma_{\text {sy }}^{2}$ & 7.4981 & 1.5280 & 7.6598 & 1.5601 \\
\hline & & $\sigma_{\varepsilon}^{2}$ & -0.4060 & 0.0548 & 2.8920 & 0.2991 \\
\hline
\end{tabular}
Models with $\mathrm{N}=49, \mathrm{~T}=10$ and $\sigma_{\mathrm{U}}{ }^{2}=0.3$

Table 2. RelBias and RMSE of Regression Coefficients and Variance Components for Bayesian Spatio-Temporal Measurement Error and Naïve

\begin{tabular}{|c|c|c|c|c|c|c|}
\hline \multicolumn{7}{|c|}{ Model } \\
\hline \multirow[t]{2}{*}{$\rho_{\mathrm{T}}$} & \multirow{2}{*}{$\left(\rho_{\mathrm{sy}}, \rho_{\mathrm{sx}}\right)$} & \multirow[t]{2}{*}{ Parameter } & \multicolumn{2}{|l|}{$\mathrm{ME}$} & \multicolumn{2}{|l|}{ NAIVE } \\
\hline & & & RelBias & $R M S E$ & RelBias & $R M S E$ \\
\hline \multirow[t]{5}{*}{0.5} & $(0.5,0.1)$ & $\beta_{0}$ & -0.0033 & 0.0530 & -0.0031 & 0.0530 \\
\hline & & $\beta_{\mathrm{x}}$ & -0.0754 & 0.2346 & -0.4530 & 0.9068 \\
\hline & & $\sigma_{s y}^{2}$ & 1.3159 & 0.3016 & 1.5657 & 0.3424 \\
\hline & & $\sigma_{\varepsilon}^{2}$ & -0.0232 & 0.0518 & 5.4041 & 0.5437 \\
\hline & $(0.5,0.5)$ & $\beta_{0}$ & -0.0080 & 0.0608 & -0.0078 & 0.0608 \\
\hline
\end{tabular}
Models with $\mathrm{N}=100, \mathrm{~T}=10$ and $\sigma_{\mathrm{U}}^{2}=0.3$ 
Table 2, cont.

\begin{tabular}{|c|c|c|c|c|c|c|}
\hline & $(0.5,0.9)$ & $\begin{array}{c}\beta_{\mathrm{x}} \\
\sigma_{\text {sy }}^{2} \\
\sigma_{\varepsilon}^{2} \\
\beta_{0} \\
\beta_{\mathrm{x}} \\
\sigma_{\mathrm{sy}}^{2} \\
\sigma_{\varepsilon}^{2} \\
\end{array}$ & $\begin{array}{l}-0.1961 \\
2.2850 \\
-0.1404 \\
0.0072 \\
-0.3154 \\
3.5928 \\
-0.1975 \\
\end{array}$ & $\begin{array}{l}0.4290 \\
0.4790 \\
0.0342 \\
0.1157 \\
0.6840 \\
0.7382 \\
0.0337 \\
\end{array}$ & $\begin{array}{l}-0.5378 \\
2.4644 \\
3.6768 \\
0.0070 \\
-0.5533 \\
3.6444 \\
2.4212 \\
\end{array}$ & $\begin{array}{l}1.0764 \\
0.5139 \\
0.3751 \\
0.1139 \\
1.1081 \\
0.7745 \\
0.2841 \\
\end{array}$ \\
\hline 0.9 & $\begin{array}{l}(0.5,0.5) \\
(0.5,0.9)\end{array}$ & $\begin{array}{c}\beta_{0} \\
\beta_{\mathrm{x}} \\
\sigma_{\mathrm{sy}}^{2} \\
\sigma_{\varepsilon}^{2} \\
\beta_{0} \\
\beta_{\mathrm{x}} \\
\sigma_{\mathrm{sy}}^{2} \\
\sigma_{\varepsilon}^{2} \\
\beta_{0} \\
\beta_{\mathrm{x}} \\
\sigma_{\mathrm{sy}}^{2} \\
\sigma_{\varepsilon}^{2}\end{array}$ & $\begin{array}{l}0.0143 \\
-0.2261 \\
7.9027 \\
-0.7371 \\
-0.0125 \\
-0.2808 \\
7.4721 \\
-0.6466 \\
-0.0311 \\
-0.3498 \\
7.6313 \\
-0.6241\end{array}$ & $\begin{array}{l}0.1087 \\
0.4617 \\
1.6105 \\
0.0756 \\
0.1279 \\
0.5676 \\
1.5079 \\
0.0703 \\
0.2651 \\
0.7059 \\
1.5422 \\
0.0709\end{array}$ & $\begin{array}{l}0.0144 \\
-0.4383 \\
7.9853 \\
4.7356 \\
-0.0125 \\
-0.5291 \\
7.5494 \\
3.6836 \\
-0.0310 \\
-0.5480 \\
7.7089 \\
2.9474\end{array}$ & $\begin{array}{l}0.1087 \\
0.8783 \\
1.6269 \\
0.4767 \\
0.1278 \\
1.0593 \\
1.5229 \\
0.3714 \\
0.2649 \\
1.0971 \\
1.5576 \\
0.2981\end{array}$ \\
\hline
\end{tabular}

Tables 3 show the overall fit statistics for the Spatio-Temporal Measurement Error and Naïve Models. The MLIK, DIC, and WAIC all tend to favor the Spatio-Temporal Measurement Error model for all sample sizes $(N)$ and for all combination the spatial and temporal dependence parameter. The percentage $(\%)$ of samples that the criteria choose the Spatio-Temporal Measurement Error model as the best model are $100 \%$.

Table 3. MLIK, DIC and WAIC of Bayesian Spatio-Temporal Measurement Error and Naïve Models.

\begin{tabular}{|c|c|c|c|c|c|}
\hline \multicolumn{6}{|c|}{ Model } \\
\hline $\mathrm{N}$ & $\rho_{\mathrm{T}}$ & $\begin{array}{l}\left(\rho_{\mathrm{sy}},\right. \\
\left.\rho_{\mathrm{sx}}\right)\end{array}$ & Criterion & ME & NAIVE \\
\hline 49 & 0.5 & $\begin{array}{l}(0.5, \\
0.1) \\
\\
(0.5, \\
0.5) \\
\\
(0.5, \\
0.9)\end{array}$ & $\begin{array}{l}\text { MLIK } \\
\text { DIC } \\
\text { WAIC } \\
\text { MLIK } \\
\text { DIC } \\
\text { WAIC } \\
\text { MLIK } \\
\text { DIC } \\
\text { WAIC } \\
\end{array}$ & $\begin{array}{l}-1487.77(100 \%) \\
629.84(100 \%) \\
544.15(100 \%) \\
-1431.31(100 \%) \\
557.49(100 \%) \\
462.50(100 \%) \\
-1453.38(100 \%) \\
559.31(100 \%) \\
486.05(100 \%) \\
\end{array}$ & $\begin{array}{l}-863.83(0 \%) \\
1299.21(0 \%) \\
1305.52(0 \%) \\
-839.28(0 \%) \\
1211.97(0 \%) \\
1217.85(0 \%) \\
-845.03(0 \%) \\
1147.52(0 \%) \\
1144.70(0 \%) \\
\end{array}$ \\
\hline & 0.9 & $\begin{array}{l}(0.5, \\
0.1) \\
\\
(0.5, \\
0.5) \\
\\
(0.5, \\
0.9)\end{array}$ & $\begin{array}{l}\text { MLIK } \\
\text { DIC } \\
\text { WAIC } \\
\text { MLIK } \\
\text { DIC } \\
\text { WAIC } \\
\text { MLIK } \\
\text { DIC } \\
\text { WAIC }\end{array}$ & $\begin{array}{l}-1661.14(100 \%) \\
527.07(100 \%) \\
437.23(100 \%) \\
-1582.86(100 \%) \\
531.94(100 \%) \\
480.21(100 \%) \\
-1611.37(100 \%) \\
530.89(100 \%) \\
485.20(100 \%)\end{array}$ & $\begin{array}{l}-908.28(0 \%) \\
1289.97(0 \%) \\
1296.54(0 \%) \\
-878.62(0 \%) \\
1208.86(0 \%) \\
1214.10(0 \%) \\
-877.82(0 \%) \\
1161.82(0 \%) \\
1163.01(0 \%)\end{array}$ \\
\hline 100 & 0.5 & $\begin{array}{l}(0.5, \\
0.1) \\
\\
(0.5, \\
0.5) \\
\\
(0.5, \\
0.9)\end{array}$ & $\begin{array}{l}\text { MLIK } \\
\text { DIC } \\
\text { WAIC } \\
\text { MLIK } \\
\text { DIC } \\
\text { WAIC } \\
\text { MLIK } \\
\text { DIC } \\
\text { WAIC } \\
\end{array}$ & $\begin{array}{l}-3007.74(100 \%) \\
1188.50(100 \%) \\
1061.96(100 \%) \\
-2887.13(100 \%) \\
1270.71(100 \%) \\
1245.92(100 \%) \\
-2903.56(100 \%) \\
1151.80(100 \%) \\
1078.52(100 \%)\end{array}$ & $\begin{array}{l}-1751.40(0 \%) \\
2639.90(0 \%) \\
2652.42(0 \%) \\
-1699.7(0 \%) \\
2446.95(0 \%) \\
2457.80(0 \%) \\
-1693.20(0 \%) \\
2255.15(0 \%) \\
2240.24(0 \%) \\
\end{array}$ \\
\hline & 0.9 & $\begin{array}{l}(0.5, \\
0.1) \\
(0.5, \\
0.5) \\
\\
(0.5, \\
0.9)\end{array}$ & $\begin{array}{l}\text { MLIK } \\
\text { DIC } \\
\text { WAIC } \\
\text { MLIK } \\
\text { DIC } \\
\text { WAIC } \\
\text { MLIK } \\
\text { DIC } \\
\text { WAIC }\end{array}$ & $\begin{array}{l}-3362.06(100 \%) \\
799.02(100 \%) \\
542.76(100 \%) \\
-3188.09(100 \%) \\
759.13(100 \%) \\
564.58(100 \%) \\
-3209.56(100 \%) \\
857.76(100 \%) \\
660.73(100 \%)\end{array}$ & $\begin{array}{l}-1839.56(0 \%) \\
2631.99(0 \%) \\
2642.410 \%) \\
-1780.38(0 \%) \\
2471.31(0 \%) \\
2478.72(0 \%) \\
-1767.71(0 \%) \\
2373.18(0 \%) \\
660.73(0 \%) \\
\end{array}$ \\
\hline
\end{tabular}

\section{Conclusion}

In this paper, we investigate the bias induced in the estimated regression coefficient when covariates are measured with error in spatio-temporal regression modeling using Bayesian approach. We consider different spatial and temporal dependence parameter of response and covariate.

The simulation results show that the naïve Bayesian analysis that ignores measurement error will attenuate estimated regression coefficient towards the null. Furthermore, we observe that the amount of attenuation increase with the spatial dependence parameter of covariate, but decrease with the temporal dependence parameter. In contrast, the Bayesian analysis considering measurement error show more accurate and efficient estimated regression coefficient compared with naïve analysis.

\section{REFERENCES}

[1] L. Anselin,. Spatial Econometrics. A Companion to Theoretical Econometrics. Edited by Badi H. Baltagi. Blackwell Publishing Ltd, 2003.

[2] J. P. LeSage, 2014. Spatial econometrics panel data model specification: A Bayesian approach. Spatial Statistics, 9, 122-145.

[3] L. Bernardinelli, C. Pascutto, N. G. Best, and W. R. Gilks. 1997. Disease mapping with errors in covariates. Statist. Medicine, 16, 741-752.

[4] Y. Li, H. Tang, X. Lin, 2009. Spatial linear mixed models with covariate measurement errors. Statistica Sinica 19(3): 1077 .

[5] Huque, M. H., H.D Bondell, and L. Ryan. 2014. On the impact of covariate measurement error on spatial regression modelling. Environmetrics 25:560-570.

[6] Muff, S., A. Riebler, L. Held, H. Rue, and P. Saner. 2015. Bayesian analysis of measurement error models using integrated nested Laplace approximations. J. R. Stat. Soc. Ser. C. Appl. Stat. 64 (2): 231-252.

[7] Stoklosa J., P. Dann, R. M. Huggins and W. H. Hwang. 2016. Estimation of survival and capture probabilities in open population capture-recapture models when covariates are subject to measurement error. Computational Statistics and Data Analysis 96, 74-86.

[8] Huque, M. H., H.D. Bondell, R. J. Carroll and L. Ryan. 2016. Spatial regression with covariate measurement error: A semiparametric approach. Biometrics:1-9.

[9] Xia, H. and B. P Carlin. 1998. Spatio-temporal models with errors in covariates: mapping Ohio lung cancer mortality. Statist. Medicine 17, 2025-2043.

[10] Rushworth A., D. Lee and R. Mitchell. 2014. A spatio-temporal model for estimating the long-term effects of air pollution on respiratory hospital admissions in Greater London. Spatial and Spatio-temporal Epidemiology 10:29-38.

[11] Truong L. T., L. Kieu and T. A. Vu. 2016. Spatio-temporal and random parameter panel data models of traffic crash fatalities in Vietnam. Accident Analysis and Prevention 94:153-161. 
[12] Gelman, A., Carlin J. B., Stern H. S., Dunson D. B., Vehtari A, and Rubin, D.B., Bayesian Data Analysis. Chapman \& Hall/CRC, New York, NY, 2014. 